\title{
MDM2 antagonist Nutlin-3a potentiates antitumour activity of cytotoxic drugs in sarcoma cell lines
}

\author{
Hege O Ohnstad ${ }^{1}$, Erik B Paulsen ${ }^{1}$, Paul Noordhuis ${ }^{1}$, Marianne Berg ${ }^{2,3}$, Ragnhild A Lothe ${ }^{2,3}$, Lyubomir T Vassilev ${ }^{4}$ \\ and Ola Myklebost ${ }^{1,5^{*}}$
}

\begin{abstract}
Background: Frequent failure and severe side effects of current sarcoma therapy warrants new therapeutic approaches. The small-molecule MDM2 antagonist Nutlin-3a activates the p53 pathway and efficiently induces apoptosis in tumours with amplified MDM2 gene and overexpression of MDM2 protein. However, the majority of human sarcomas have normal level of MDM2 and the therapeutic potential of MDM2 antagonists in this group is still unclear. We have investigated if Nutlin-3a could be employed to augment the response to traditional therapy and/or reduce the genotoxic burden of chemotherapy.
\end{abstract}

Methods: A panel of sarcoma cell lines with different TP53 and MDM2 status were treated with Nutlin-3a combined with Doxorubicin, Methotrexate or Cisplatin, and their combination index determined.

Results: Clear synergism was observed when Doxorubicin and Nutlin-3a were combined in cell lines with wildtype TP53 and amplified MDM2, or with Methotrexate in both MDM2 normal and amplified sarcoma cell lines, allowing for up to tenfold reduction of cytotoxic drug dose. Interestingly, Nutlin-3a seemed to potentiate the effect of classical drugs as Doxorubicin and Cisplatin in cell lines with mutated TP53, but inhibited the effect of Methotrexate.

Conclusion: The use of Nutlin in combination with classical sarcoma chemotherapy shows promising preclinical potential, but since clear biomarkers are still lacking, clinical trials should be followed up with detailed tumour profiling.

\section{Background}

The TP53 gene, coding for the transcription factor p53, is thought to be the most frequently mutated gene in cancer, inactivated in about $50 \%$ of all tumours. However, aberrations of this pathway are probably even more widespread, as tumours retaining wild-type p53 $\left(T P 53^{W t}\right)$ might have defects in other parts of the pathway [1]. In sarcomas, malignant tumours resembling mesenchymal tissue, amplification of $M D M 2$ (murine double minute 2) is relatively common (20\%) in tumours having $T P 53^{W t}$, resulting in disabled p53 function because overexpressed MDM2 protein binds to and inactivates p53 [2,3].

\footnotetext{
* Correspondence: ola.myklebost@imbv.uio.no

'Department of Tumour Biology, The Norwegian Radium Hospital, Oslo

University Hospital, P O Box 4953 Nydalen, NO-0424 Oslo, Norway

Full list of author information is available at the end of the article
}

Remaining tumours may have other aberrations in their p53 pathway, either p53 mutations (TP53 ${ }^{\text {Mut }}, 11-31 \%$ depending on subtype), or other changes in the downstream pathway that do not affect the level of MDM2 (MDM2 $2^{W t} / T P 53^{W t}, 11-88 \%$ depending on subtype) [4-6].

Sarcomas are among the more frequent cancers among children [7], and both children and adults are treated with intensive surgery, chemotherapy or radiation, or a combination of these. Currently used chemotherapy (e.g. Methotrexate, Cisplatin and Doxorubicin) is frequently inadequate, with $50-80 \%$ long-term survival depending on tumour subgroup [8,9] and associated with severe toxicity. Due to the frequent failure of prevailing therapy and unacceptable adverse effects there is an urgent need for new therapeutic modalities in sarcoma.

\section{() Biomed Central}


Nutlin-3a, a novel small-molecule inhibitor of the p53MDM2 interaction, has been shown to be highly effective in killing osteosarcoma cells in vitro and reducing tumour burden in vivo $[10,11]$. Nutlin-3a displaces p53 from the binding pocket of MDM2 and thereby releases p53 from inhibition and proteasomal degradation, leading to induction of its downstream targets, cell cycle arrest, and apoptosis. Tumours with amplification of the $M D M 2$ gene $\left(M D M 2^{A m p l}\right)$ are most responsive to Nutlin, most likely due to otherwise intact downstream p53 signalling [11]. However, the apoptotic response in cancer cells with normal levels of MDM2 can vary dramatically, suggesting that other mechanisms or modifying factors are involved in the response to MDM2 antagonists. Several studies have suggested both p53related and unrelated factors, such as caspases, BAX, PUMA, p73 and other apoptotic factors to be involved [10,12-14], and the status of the related MDM4 (also known as $M D M X)$ gene has also been proposed to explain the responses to Nutlin [15-17].

Early studies suggest that MDM2 antagonists may be particularly effective in sarcomas because $M D M 2$ is frequently amplified in these tumours $[2,5,18,19]$. We previously confirmed the effectiveness of Nutlin-3a as a single agent in $T P 53^{W t} / M D M 2^{A m p l}$ liposarcomas [20]. However, since many sarcomas are $T P 53^{W t}$ but do not have amplified $M D M 2$ (are $M D M 2^{W t}$ ), it would be of interest to investigate if Nutlin-3a could potentiate the response of $M D M 2^{W t} / T P 53^{W t}$ tumours to conventional chemotherapy. Since p53 mutations are very heterogeneous, different mutations sites could also imply different response to combined therapy. Nutlin has been shown to be synergistic with genotoxic drugs (e.g. Fludarabine, Chlorambucil, Doxorubicin, Etoposide, Melphalan and Cisplatin) in haematological malignancies, lymphoma, neuroblastoma and hepatocellular carcinoma, and with radiation in lung cancer [12,21-26], but antagonistic with antimitotic agents (e.g. Paclitaxel) in colon cancer cell lines [27]. Paclitaxel was shown to be synergistic in rhabdomyosarcoma cell lines [28]. Here, we investigate the effect of Nutlin-3a on sarcoma cell lines in combination with the current standard of therapy (e.g. Doxorubicin, Cisplatin and Methotrexate). These are well known and frequently used genotoxic drugs that induce cell cycle arrest and apoptosis through both p53-dependent and-independent mechanisms [29-31]. The latter group is represented by Methotrexate, which primarily inhibits dihydrofolate reductase (DHFR), but also glycinamide ribonucleotide formyltransferase (GARFT) and thymidylate synthetase (TS), all key components of nucleotide biosynthesis. Our studies show significant potentiation and/or reduction of effective dose of cytotoxic drugs by Nutlin, in both wild-type and mutated TP53 tumours, suggesting that clinical combination studies in sarcoma are warranted.

\section{Methods}

\section{Cell lines and culture conditions}

We selected 5 different sarcoma cell lines, three with TP5 $3^{W t}$ and two with TP53 $3^{M u t}$. Two cell lines had $M D M 2^{A m p l}$ and three had $M D M 2^{W t}$ (Table 1). The cell line T778 (94778), kindly provided by Dr Florence Pedeutour, was established from a relapsed liposarcoma at Hopital de l'Arche [32]. Dr. A Thomas Look at St Jude's hospital, Memphis, USA, kindly donated the cell lines RMS13 [33] and OSA (available from the ATCC, Rockville, MD as SJSA-1 or CRL2098). SaOS-2 (HTB85) and U2OS (HTB96) were purchased from the ATCC. All cell lines were grown in RPMI 1640 medium (Bio Whittaker, Verviers Belgium) containing $20 \mathrm{mM}$ HEPES and 2 mM GlutaMax (GIBCO BRL Life Technologies, Paisley UK), $50 \mathrm{IE} / \mathrm{ml}$ penicillin, $0,1 \mu \mathrm{g} / \mathrm{ml}$ streptomycin (Bio Whittaker, Verviers Belgium) and 8\% heat-inactivated fetal bovine serum (PAA Laboratories, Pasching, Austria) in an environment containing $5 \% \mathrm{CO}_{2}$ at $37^{\circ} \mathrm{C}$.

\section{Direct sequencing mutation analysis of TP53}

Genomic DNA was extracted from cell lines using the standard phenol chloroform method. The total protein coding region of TP53 (exon 2 to 11) was amplified in five distinct fragments by using flanking intronic primers with M13 tails [34], generating products of 837 , 464, 887, 300 and 275 base pairs respectively. In short,

Table 1 Cell line characteristics

\begin{tabular}{|c|c|c|c|c|c|c|c|}
\hline Cell line & Histology & Patient sex/age & Origin & Site & TP53 status & MDM2 copy number & MDM2 mRNA level \\
\hline T778 & DDLS & $F / 69$ & Relapse & Retroperitoneum & wt & $59.8 \pm 1.8$ & $28.5 \pm 1.6$ \\
\hline OSA (SJSA-1) & OS & $M / 19$ & Primary & Femur & wt & $49.1 \pm 1.1$ & $43.2 \pm 9.4$ \\
\hline U2OS & OS & $F / 15$ & Primary & Tibia & wt & $0.7 \pm 0.1$ & $3.1 \pm 0.2$ \\
\hline RMS13 & RMS & $M / 17$ & Primary & Bone marrow & $\begin{array}{c}\text { mut } \\
(\text { exon 8) }\end{array}$ & $1.9 \pm 0.2$ & $0.9 \pm 0$ \\
\hline $\mathrm{SaOS}-2$ & OS & $F / 11$ & Unknown & Unknown & del & $1.4 \pm 0.2$ & $2.5 \pm 0.2$ \\
\hline
\end{tabular}

Panel of cell lines tested. DDLS refers to de-differentiated liposarcoma, OS osteosarcoma and RMS rhabdomyosarcoma. RMS13 was initially classified TP53 ${ }^{W t}$, but reclassified as TP53 ${ }^{\text {Mut }}$ after extended DNA sequence analysis. MDM2 copy number and expression were determined previously as described in Müller et al, 2007. 
all the five fragments were amplified in separate PCR reactions using identical conditions for each primer pair. We used HotStar Polymerase (Qiagen, GmbH, Germany) for PCR. The quality and quantity of the resulting PCR product was evaluated on a polyacrylamide gel, and the product was purified using ExoSAP-IT (USB Corporation, Cleveland, Ohio, USA). Both 5' and 3' sequencing reactions were performed using BigDye terminator v3.1 kit from Applied Biosystems (Foster City, California, USA) and adding M13 primers to the PCR product. The resulting sequence product was further purified using Millipore multiscreen plates (Millipore, Billerica, Massachusetts, USA) with Sephadex G-50 Superfine (GE Healthcare, Chalfont St. Giles, United Kingdom), and subjected to sequencing on a 3730 DNA Analyzer (Applied Biosystems). Where a mutation was detected, a new independent PCR product was subjected to sequencing to confirm our findings.

\section{Drug sensitivity assay}

Cellular integrity was measured as total cellular protein by the Sulforhodamine B (SRB) assay, which was performed essentially as described previously [35]. Briefly, in a 96-well plate (Becton Dickinson), cells were seeded in $100 \mu \mathrm{l} /$ well at appropriate cell densities; 500 cells per well for OSA, 1500 for T778, 10000 for RMS13, 2000 for U2OS and 8000 for SaOS-2. At these densities the untreated control cells were growing exponentially during the entire incubation period. After 24 hours, $100 \mu \mathrm{l}$ drug-containing medium was added and the cells were cultured for another 120 hours.

All cell lines were exposed to the drugs alone or in combination. The IC50 of the individual drug was determined where after combinations were made based on the ratio of the IC50's. Drugs were added in a constant ratio design. For Doxorubicin and Cisplatin three fixed ratios were set: Ratio $1: 1=\mathrm{IC}_{5} 0_{\text {Nutlin-3a }}$ IC $50_{\mathrm{x}}$, ratio $1: 2$ $=I C 50_{\text {Nutlin-3a }}: 2$ times $I C 50_{\mathrm{x}}$ and ratio $2: 1=2$ times IC $50_{\text {Nutlin-3a: }}$ IC $50_{x}$, where $\mathrm{x}$ is the chemotherapy. For Methotrexate we used the 1:1 ratio only. When the ratio was set, a mixture of the two drugs was made and serially diluted to obtain a good dosage range.

After exposure, cells were fixed with 50\% Trichloroacetic acid (TCA, Sigma-Aldrich, $25 \mu \mathrm{l} /$ well), washed and stained with $0.4 \%$ Sulforhodamine B (SRB, SigmaAldrich) dissolved in 1\% acetic acid (Merck, $50 \mu \mathrm{l} /$ well), subsequently washed five times with $1 \%$ acetic acid to remove unbound stain. Protein bound stain was dissolved in $150 \mu \mathrm{l} 10 \mathrm{mM}$ Tris base (Merck). Optical density was measured at $540 \mathrm{~nm}$ in a Victor Wallac 1420 multi-label counter (Perkin Elmer, MA USA). Relative Growth was calculated as follows:

$$
\left(\left(\mathrm{OD}_{\text {treated }}-\mathrm{OD}_{\text {zero }}\right) /\left(\mathrm{OD}_{\text {control }}-\mathrm{OD}_{\text {zero }}\right)\right) \times 100 \%
$$

when $\mathrm{OD}_{\text {treated }}-\mathrm{OD}_{\text {zero }}>0$; Cell death occurred if $\mathrm{OD}_{\text {treated }}-\mathrm{OD}_{\text {control }}<0$, where $\mathrm{OD}_{\text {treated }}$ represents the optical density of treated cells at the day of the assay, $\mathrm{OD}_{\text {control }}$ the optical density of the untreated control cells and $\mathrm{OD}_{\text {zero }}$ the optical density at the moment of drug addition. From the relative growth we obtain the fraction affected $(\mathrm{Fa})$ as follows:

$$
1-\left(\left(\mathrm{OD}_{\text {treated }}-\mathrm{OD}_{\text {zero }}\right) /\left(\mathrm{OD}_{\text {control }}-\mathrm{OD}_{\text {zero }}\right)\right) \text {. }
$$

\section{Combination analysis}

To evaluate the pharmacological interaction of the different combinations of drug treatments, we followed the method proposed by Chou et al. [36] Briefly, synergism, additivity or antagonism for the different combinations was calculated on the basis of the multiple drug-effect equation and quantitated by the combination index (CI) where $\mathrm{CI}=1$ indicates that the two drugs have additive effect, CI $<1$ more than additive effect ("synergism") and CI $>1$ less than additive effect ("antagonism"). The involved drugs were assumed mutually non-exclusive meaning that they have totally independent modes of action and the CI was calculated using the CalcuSyn software based on:

$$
\mathrm{CI}=(\mathrm{D})_{1} /\left(\mathrm{D}_{\mathrm{x}}\right)_{1}+(\mathrm{D})_{2} /\left(\mathrm{D}_{\mathrm{x}}\right)_{2}+\left(\mathrm{D}_{1}\right)\left(\mathrm{D}_{2}\right) /\left(\mathrm{D}_{\mathrm{x}}\right)_{1}\left(\mathrm{D}_{\mathrm{x}}\right)_{2}
$$

where $\left(D_{x}\right)_{1}$ and $\left(D_{x}\right)_{2}$ are the doses of drug 1 and drug 2 alone inhibiting $\mathrm{x} \%$ whereas $\left(D_{1}\right)$ is the dose of drug 1 in combination with drug 2 and $\left(D_{2}\right)$ the dose of drug 2 in combination with drug 1 that gives the experimentally observed $x$ inhibition. Since our aim was to achieve maximal effect of the drugs tested on cancer cells, a mean CI was calculated from data points with fraction affected $(\mathrm{Fa})>0.5$. $(\mathrm{Fa})<0.5$ would imply lower growth inhibition and a large fraction of the cell population would still grow. Fa $<0.5$ was therefore considered not relevant. Furthermore we evaluated how much each drug dose in a synergistic combination could be reduced at a given effect level compared with the doses for each drug alone, the so-called dose-reduction index (DRI):

$$
(\mathrm{DRI})_{1}=\left(\mathrm{D}_{\mathrm{x}}\right)_{1} /(\mathrm{D})_{1} \text { and }(\mathrm{DRI})_{2}=\left(\mathrm{D}_{\mathrm{x}}\right)_{2} /(\mathrm{D})_{2}
$$

Notably, non-synergistic combinations could also result in reduced drug doses (DRI $>1$ ) as illustrated by the example: if drug $\mathrm{A}$ and drug $\mathrm{B}$ each inhibit $50 \%$ and if $(0.5 \mathrm{~A}+0.5 \mathrm{~B})$ also inhibits $50 \%$ and both drugs have no overlapping toxicity toward the host, DRI $\geq 1$ may still be beneficial [37].

\section{Immunoblotting}

In the drug sensitivity assay we chose a constant ratio design with three fixed ratios as described above. By only including the 1:1 ratio in the western analysis, our 
intent was to eliminate the potential disturbing effect of different drug concentrations on the molecular mechanisms. Cells were treated for 24 hours with Nutlin-3a, Doxorubicin, Cisplatin, Methotrexate or described drug combinations. They were lysed in lysis buffer (supplemented with phosphatase and protease inhibitor cocktails (Sigma-Aldrich)) and sonicated. 15$30 \mu \mathrm{g}$ of protein lysate was separated on $4-12 \%$ NuPage BIS-Tris gradient gels (Invitrogen, CA USA) and transferred to $0.45 \mu \mathrm{m}$ PVDF membranes (Millipore Corp., MA USA) in blotting buffer with $20 \%(\mathrm{v} / \mathrm{v})$ methanol using wet blot equipment from BIO-RAD, CA USA. Antibodies used (monoclonal except when noted): anti-p53 (1:3000 Santa Cruz sc-6243), antiMDM2 (1:300, Chemicon MAB1434), anti-MDM4 (1:20000 Bethyl Labs), anti-PUMA (1:5000, Abcam), anti- $\beta$-actin (1:4000, Sigma-Aldrich), and polyclonal anti-p21 (1:300, Santa Cruz sc-397). All HRP-conjugated secondary antibodies were from Dako, Denmark. Bands were visualized using SuperSignal West Dura ECL (\# 34076, Pierce, IL USA) and Kodak X-VIS film and software (Eastman Kodak, NY USA). Pictures were merged and presented using Photoshop (Adobe Systems, USA).

\section{Statistics}

The Statistical package SPSS 13.0 was used. 2-tailed unpaired Student's T-test on transformed results was used for statistical analysis. Changes were considered to be significant when $P<0.05$.

\section{Results}

We investigated the effect of combining Nutlin-3a with the current standard of sarcoma therapy (Doxorubicin, Cisplatin, Methotrexate) on the growth of five sarcoma cell lines. The characteristics and sources of the cell lines used are described in Table 1. From our previous results we knew that the liposarcoma cell line T778 was sensitive to Nutlin-3a and we included the osteosarcoma cell line OSA with similar characteristics ( $M D M 2^{A m p l} /$ $T P 53^{W t}$ ) as a control cell line. As representative of $M D M 2^{W t} / T P 53^{W t}$, we chose the osteosarcoma cell line U2OS. Finally, SaOS-2 and RMS13 were chosen as examples of two different cell lines with mutated p53. SaOS-2 is known to be TP53 $3^{\text {Null }}$ [38]. RMS13, previously described as TP53 ${ }^{W t}$, did not respond to Nutlin-3a, and was found to have a transversion in codon 280 , exon 8 , changing the amino acid from arginine to serine in the resulting protein. Protein analysis uncovered high levels of probably dysfunctional p53 protein and constitutively high p21 and PUMA (see below). This is consistent with a report that the Rh30 cell line, established independently from a xenograft from the same patient, also had this mutation [39] and illustrates the usefulness of
Nutlin as a probe for p53 status. The other cell lines with TP53 $3^{W t}$ were validated by sequencing.

\section{Single drug treatment}

The effect of each drug alone is presented in Table 2. The $M D M 2^{A m p l}$ cell lines (OSA and T778) were sensitive to Nutlin-3a and showed clear dose dependence, whereas the MDM2 $2^{W t} / T P 53^{W t} \mathrm{U} 2 \mathrm{OS}$ was less sensitive, with an IC50 about twice that for the $M D M 2^{A m p l}$ cell lines. Consistent with previous results, we were not able to reach $50 \%$ growth inhibition for the TP53 ${ }^{M u t}$ cell lines (SaOS-2 and RMS13) when using Nutlin-3a alone. Cisplatin, Doxorubicin and Methotrexate also inhibited cell growth in a concentration-dependent manner. Figure 1 shows representative growth inhibition curves for the $M D M 2^{A m p l} / T P 53^{W t}$ OSA and MDM $2^{W t} / T P 53^{M u t}$ RMS13 exposed to single drugs. In all cell lines tested, Doxorubicin and Methotrexate were more cytotoxic than Cisplatin or Nutlin-3a when used alone (Table 2), and Methotrexate was most effective in the $M D M 2^{A m p l}$ cell lines. Doxorubicin on the other hand was most cytotoxic in the TP53 ${ }^{M u t}$ line, RMS13. For Cisplatin, the most sensitive cell line tested was OSA, followed by RMS13, U2OS, T778 and SaOS-2.

\section{Combination treatments}

Multiple drug-effect analysis was performed for combinations of Nutlin-3a with Doxorubicin, Cisplatin or Methotrexate using the CalcuSyn software. Fraction affected $(\mathrm{Fa})$ values (indicating the fraction of cells inhibited after drug exposure) were obtained after exposure of the cells to a series of drug concentrations. To indicate the effects at different Fa values, combination index (CI) values were calculated for each Fa. For the TP53 ${ }^{\text {Mut }}$ cell lines RMS13 and SaOS-2 we were not able to calculate any combination effect since the cell lines did not respond to Nutlin-3a. Figure 2 shows an Fa-CI plot illustrating the effects of Nutlin-3a and Doxorubicin in a 1:1 fixed drug ratio combination and demonstrates synergism at $\mathrm{Fa}>0.5$ for T778 whereas on average the other two cell lines display less than additive effects. As

Table 2 Growth inhibition by Nutlin-3a, Doxorubicin, Cisplatin and Methotrexate for the sarcoma cell lines

\begin{tabular}{lccccc}
\hline \multicolumn{5}{c}{ Mean IC50 \pm SE(nM) } \\
\hline Drugs & OSA & T778 & U2OS & RMS13 & SaOS-2 \\
\hline Nutlin-3a & $527 \pm 131$ & $658 \pm 138$ & $1024 \pm 485$ & na & na \\
Dox & $62 \pm 77$ & $62 \pm 31$ & $40 \pm 35$ & $14 \pm 4$ & $77 \pm 12$ \\
Cis & $758 \pm 592$ & $1566 \pm 1369$ & $1037 \pm 596$ & $855 \pm 143$ & $4828 \pm 4855$ \\
Mtx & $30 \pm 9$ & $59 \pm 74$ & $181 \pm 153$ & $106 \pm 34$ & $636 \pm 806$ \\
\hline
\end{tabular}

Cells were exposed to a concentration range of Nutlin-3a, Doxorubicin, Cisplatin and Methotrexate for $120 \mathrm{~h}$. Values (IC50 in nM) are means \pm standard error of the mean (SE) for 3-5 experiments. RMS13 and SaOS-2 were insensitive to Nutlin-3a consistent with their TP53 $3^{\text {Mut }}$. 


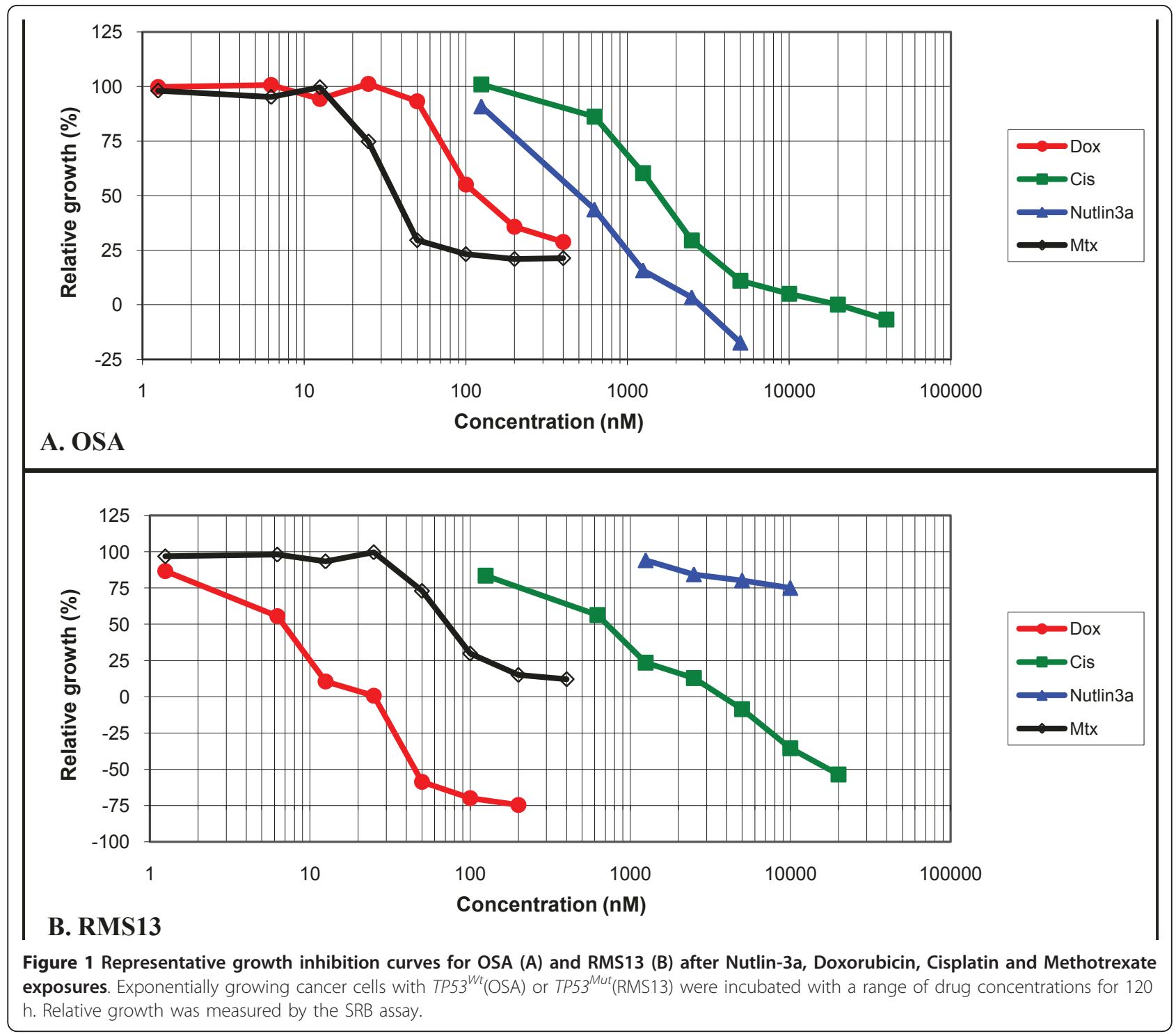

we wanted to determine the combination effect at concentrations showing efficacy, we limited the analysis to Fa greater than 0.5. The mean CI values of the various combinations for $\mathrm{Fa}>0.5$ are shown in Table 3A. Overall we found additive or more than additive effects when Nutlin-3a was combined with Doxorubicin for 4 out of 6 ratios tested for $M D M 2^{A m p l}$ cell lines, whereas Cisplatin in combination with Nutlin-3a showed less than additive effect for 5 out of 6 ratios. Nutlin-3a together with Cisplatin or Doxorubicin also showed less than additive effects in the $M D M 2^{W t}$ cell line U2OS. Methotrexate on the other hand showed more than additive effect when combined with Nutlin-3a for T778 and U2OS, $M D M 2^{A m p l}$ and $M D M 2^{W t}$, respectively.

As expected, synergism, corresponding to $\mathrm{CI}<1$, always yielded a favourable dose reduction index (DRI >
1) for both drugs. The DRI values at $\mathrm{IC}_{75}, \mathrm{IC}_{90}$ and $\mathrm{IC}_{95}$ are reported in Table 3B. These indicate that chemotherapy doses may be significantly reduced for combinations with Nutlin-3a that are synergistic. In both $M D M 2^{A m p l}$ cell lines, Doxorubicin could be reduced 10fold and in T778 the Cisplatin dose could also be significantly reduced when combined with Nutlin at a 2:1 ratio. In addition, in cell lines being $M D M 2^{A m p l}$ or $M D M 2^{W t}$, T778 and U2OS respectively, we found that reduced dose of Methotrexate would suffice when combined with Nutlin-3a. Some combinations that were not synergistic also maintained efficacy with reduced level of standard cytotoxic drugs (DRI $>1$ ) when combined with Nutlin-3a. This holds for all combinations with Doxorubicin or Methotrexate as well as some with Cisplatin. Moreover, as depicted in Table 4 Nutlin-3a clearly 
FA-Cl plot Nutlin-3a + Dox

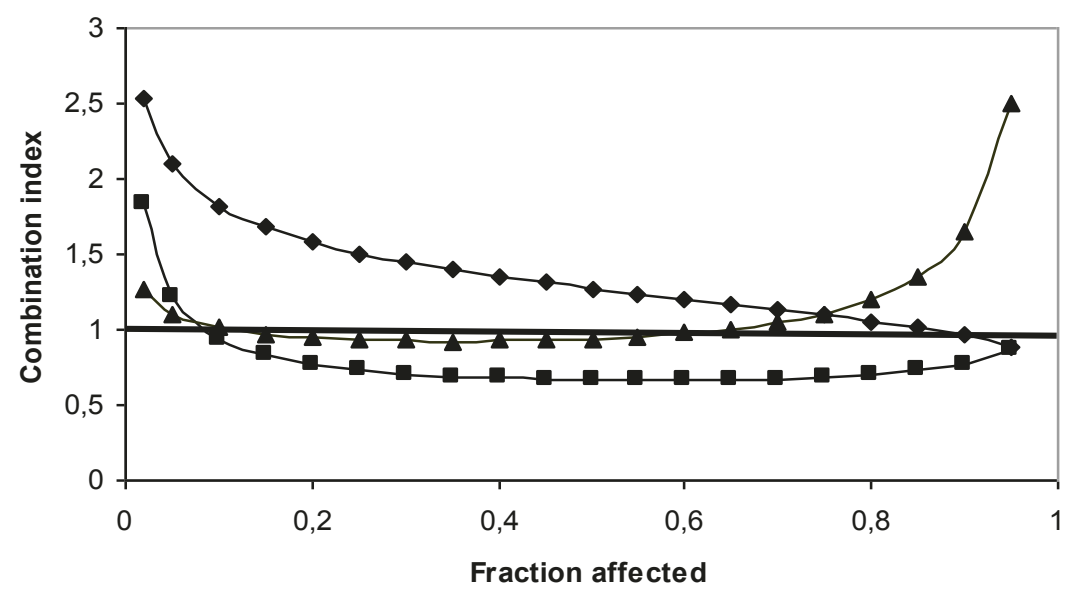

Fraction affected

Figure 2 Illustrative Fa-Cl plot for the combination of Nutlin-3a and Doxorubicin using a 1:1 fixed drug ratio. $\mathrm{Cl}$ values are calculated from each Fa for the three Nutlin-3a sensitive cell lines in the study. Here we demonstrate average synergism at Fa $>0.5$ for T778 whereas OSA and U2OS display average antagonism.

potentiated Doxorubicin and Cisplatin in RMS13 and SaOS-2 whereas Methotrexate was inhibited.

\section{Protein analysis}

Since most classic cytotoxic drugs exert their antitumour effect at least in part by activation of the p53 pathway, we investigated some key downstream p53 targets by Western blotting. As expected, Nutlin-3a induced accumulation of $\mathrm{p} 53$ protein in the $M D M 2^{A m p l}$ cell lines (Figure 3A-B and 3F), followed by activation and accumulation of p53 targets p21 and MDM2. Although the response was weaker than for Nutlin-3a alone, Doxorubicin and Cisplatin also induced p53 and its downstream targets as did the combinations. Similar to Nutlin-3a, Methotrexate induced and increased p53 in these cells, but downstream targets such as p21 and MDM2 appeared unaffected in T778. PUMA was unaffected in OSA, but induced to a similar level by all treatments in T778. The $M D M 2^{W t}$ cell line U2OS (Figure $3 \mathrm{C}$ and $3 \mathrm{~F}$ ) also displayed significant induction of p53, p21, and MDM2 after Nutlin-3a treatment alone and in combinations, whereas Cisplatin, Doxorubicin and Methotrexate alone had less effect. In this cell line, neither Nutlin-3a nor the classical drugs affected the level of PUMA, but MDM4 was completely knocked down by Methotrexate (Figure 3C and 3F), both alone and in combination with Nutlin-3a. It should be noted that $\mathrm{U} 2 \mathrm{OS}$ expressed a $62 \mathrm{kDa}$ variant of MDM4, whereas all the other lines expressed the $49 \mathrm{kDa}$ band (Figure 3). In cell lines where p53 was not functional
(RMS13 and SaOS-2), the downstream targets were not affected by any of the treatments, as expected (Figure $3 \mathrm{D}-\mathrm{F})$. Taken together we found that the combination treatments induced the $\mathrm{p} 53$ pathway proteins examined in all $T P 53^{W t}$ cell lines, whereas in $T P 53^{M u t}$ cell lines they were unaffected (Figure 3A-F).

\section{Discussion}

Current treatment options for sarcomas, based on decades of optimising high dose chemotherapy, surgery and radiation, have reached a plateau with long-term survival limited to $50-80 \%$ depending on tumour subgroup $[8,9]$. Although some new targeted treatments have been successful in other cancer types and also in some sarcoma subtypes (e.g. GIST) [40], these treatments rarely increase long-term survival. Since sarcomas are among the most frequent childhood cancers, the possibility of reducing the long-term adverse effects of high-dose chemotherapy is an important objective. Thus, new treatments targeting key cancer pathways are highly desirable. One such pathway is the p53 tumour suppressor pathway.

Although TP53 mutations are not so frequent in sarcomas [41], the pathway may also be inactivated by amplification of the $M D M 2$ gene $[2,5,18]$ which is observed almost exclusively in tumours where TP53 is wild-type. However, these two mechanisms do not account for all tumours, and although some complementary mechanisms have been suggested [6], it is still unclear if and how the pathway is inactivated in the 
Table 3 Effects of combining Nutlin-3a with chemotherapy

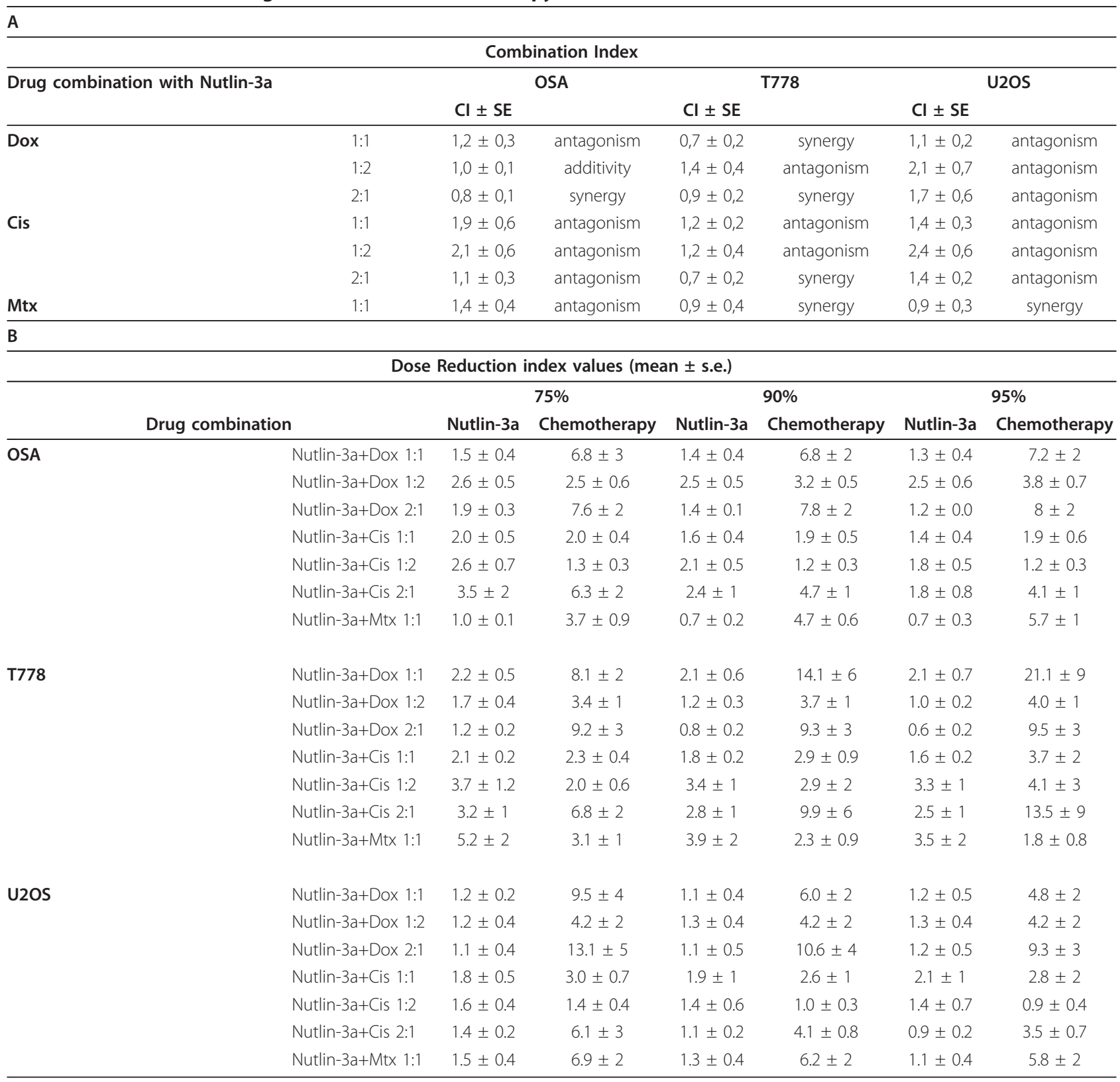

A Combination Index $(\mathrm{Cl})$ values listed in the table are means \pm standard error of the mean (SE) for Fa-values $>0.5$, indicating additivity, more than additivity (synergy) or less than additivity (antagonism). Values were determined experimentally and calculated using the Chou and Talalay method and CalcuSyn software. Relative growth was assessed $120 \mathrm{~h}$ after drug treatment. Data is shown as combinations of Nutlin-3a with Doxorubicin, Cisplatin or Methotrexate in 1:1, 1:2 and 2:1 ratios. Since Nutlin-3a is not active in RMS13 and SaOS-2 it was by definition not possible to calculate their Cl. B Dose Reduction Index (DRI) values for the three combinations at 75, 90 and 95\% levels of growth inhibition of OSA, T778 and U2OS cell growth.

$\mathrm{Cl}<1$ : more than additivity (synergy).

$\mathrm{Cl}=1$ : additivity.

$\mathrm{Cl}>1$ : less than additivity (antagonism).

remainder of sarcomas. If tumours contain defects of the p53 pathway that are upstream of p53 or suppress p53 levels, activation of p53 could bypass the defect and, provided the right signals are present, either induce apoptosis directly or sensitize the cells to induction of apoptosis by traditional therapy.
Small-molecule MDM2 antagonists such as Nutlin-3a are interesting in this aspect not only as therapeutic agents, but as tools to study p53 pathway functionality [11]. Nutlins efficiently activate p53 signalling and induce cell cycle arrest and apoptosis in sensitive cancer cells, while sparing most normal cells when tested in 
Table 4 Growth inhibition of combination treatment in TP53 ${ }^{M u t}$ cell lines

\begin{tabular}{cccccccc}
\hline & \multicolumn{3}{c}{ Mean IC50 \pm SE(nM) } \\
\hline & alone & Doxorubicin & \multicolumn{2}{c}{ Cisplatin } & \multicolumn{2}{c}{ Methotrexate } \\
& $14 \pm 4$ & $6 \pm 3$ & combination & alone & combination & alone & combination \\
\hline RMS13 & $77 \pm 12$ & $40 \pm 17$ & $485 \pm 143$ & $244 \pm 85$ & $106 \pm 34$ & $253 \pm 75$ \\
SaOS-2 & $77 \pm 4855$ & $517 \pm 229$ & $636 \pm 806$ & na \\
\hline
\end{tabular}

RMS13 and SaOS-2 cells were exposed to a concentration range of Nutlin-3a, Doxorubicin, Cisplatin, Methotrexate and combinations for $120 \mathrm{~h}$. Values (IC50 in $\mathrm{nM}$ ) are means \pm standard error of the mean (SE) for 3-5 experiments. The decrease observed in the combination of Nutlin-3a with Doxorubicin or Cisplatin, indicates that Nutlin-3a potentiates Doxorubicin and Cisplatin, whereas the increase in IC50 observed for the combination with Methotrexate indicates that Nutlin-3a inhibits Methotrexate.

vitro [10-13,15,23,27,42]. However, their safety in humans is still unknown, especially when combined with traditional cytotoxic therapy. Although it has been shown that Nutlin alone is very efficacious in $M D M 2^{A m p l}$ sarcoma cell lines in vitro and in vivo $[11,20]$, the effects of combinations with standard therapeutics in sarcomas is still unknown. The aim of this study was to investigate possible synergistic interactions between traditional sarcoma drugs and Nutlin-3a.

As expected, cell lines with wild-type TP53 responded to Nutlin-3a exposure by activation of p53 and its downstream targets, including p21 and MDM2, whereas tumours with mutated TP53, irrespective of mutation site, did not respond to Nutlin-3a alone. $M D M 2^{A m p l}$ cell lines were more sensitive to Nutlin-3a compared to the $M D M 2^{W t}$ cell lines in spite of similar levels of induction of p21, MDM2 and PUMA. This is probably the result of other yet undiscovered defects in the downstream p53 signalling in the MDM2 $2^{W t}$ cells. MDM4 is an MDM2 homologue that shares some of the same p53 binding sites as MDM2, although there are some differences [43]. It has been suggested that high levels of MDM4 may counteract Nutlin because its binding to p53 is not inhibited by the treatment [15,16,44], although the hypothesis is still controversial [45].

The genotoxic agents tested in this study are commonly used in sarcoma treatment. Doxorubicin and Methotrexate were quite effective in these cell lines, whereas higher doses of Cisplatin and Nutlin-3a were required in the TP5 $3^{W t}$ lines. Neither Doxorubicin nor Cisplatin showed consistent differences in effect in relation to MDM2 amplification or TP53 mutation, but the TP53 mutated cell line (RMS13) was more sensitive to all three genotoxic agents tested than the TP53 null cell line (SaOS-2). Methotrexate was more effective in the lines with $M D M 2$ amplification, suggesting some relation between MDM2 and the Methotrexate mechanism of action that warrants further investigation.

The DRI values clearly illustrated the benefit of the synergistic combinations, with 10 -fold reductions in Doxorubicin and Cisplatin when combined with Nutlin-3a, but also combinations with less than additive effects showed promising DRI values. Nutlin-3a and Doxorubicin or Cisplatin were less than additive in the $M D M 2^{\mathrm{Wt}} /$ TP53 ${ }^{W t} \mathrm{U} 2 \mathrm{OS}$ cell line. This is consistent with previous results in colon (HCT116) and ovarian (A2780) cancer cells, where Nutlin was cytoprotective in combination with Cisplatin [14]. Compellingly the TP5 $3^{M u t}$ cell lines, RMS13 and SaOS-2, showed a significant sensitization towards Cisplatin and Doxorubicin at IC50 when combined with Nutlin-3a. This apparently p53-independent effect of Nutlin-3a is in line with previous publications [46-48], and suggests efficacy against a wide spectrum of tumours. p73 has been proposed to be one of the factors responsible for the effect observed in TP53-null or-mutant cells. It was reported that when cells with dysfunctional TP53 were exposed to high concentrations of Nutlin (e.g. 20-30 $\mu \mathrm{M})$, p73 was displaced from its binding to MDM2, resulting in cell cycle arrest and apoptosis $[25,46,49]$. Nutlin combined with Cisplatin or Doxorubicin has also previously been reported to induce apoptosis much more efficiently than either drug alone in cells with dysfunctional TP53, most likely due to E2F1-mediated up-regulation of p73 activity [48].

The third chemotherapy tested here, Methotrexate, acts through nucleotide metabolism, for which E2F1 is a central transcription factor [50,51]. Additional targeting of the p53 pathway by Nutlin-3a could be complementary to this mode of action [52]. We found synergistic effects for both TP53 $3^{W t} / M D M 2^{\mathrm{Ampl}}$ and TP5 $3^{W t} /$ $M D M 2^{\mathrm{Wt}}$ cell lines and DRI values that implied that Methotrexate could be reduced ten-fold when combined with Nutlin-3a. In the MDM2 $2^{\mathrm{Wt}}$ cell line U2OS, treatment with Methotrexate decreased MDM4 to undetectable levels both alone and in combination with Nutlin$3 \mathrm{a}$. According to the current hypothesis, reduction of MDM4 could lead to more unbound p53 [53], but since this reduction also happened with Methotrexate only, it would not contribute to the observed synergy. On the other hand, Nutlin-3a can indirectly reduce the level of E2F1 through MDM2 mediated degradation [48], and recent results also document the reduction of DHFR through MDM2 [54]. It is tempting therefore to speculate on a double inhibition of the nucleotide metabolism when using Methotrexate together with Nutlin-3a. The inhibitory effect observed in the TP5 $53^{M u t}$ cell line 
A

\section{OSA}

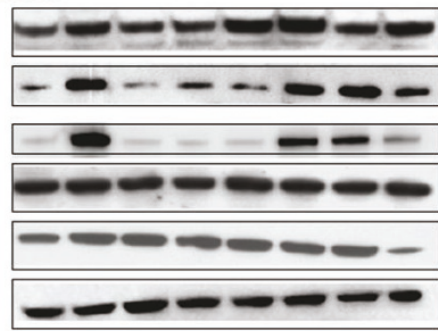

Ctrl N Cis Dox Mtx N:C N:D N:M

C

U2OS

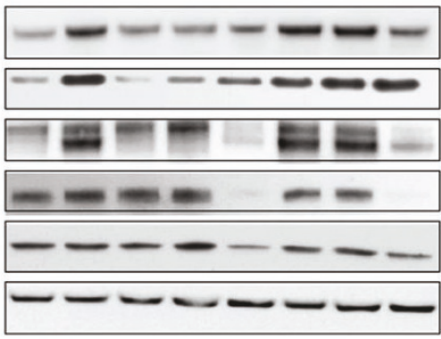

Ctrl N Cis Dox Mtx N:C N:D N:M

E

SaOS-2

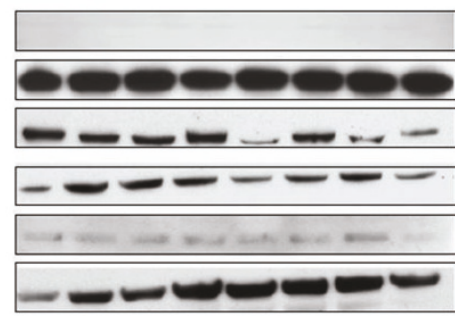

p53

p21

MDM2

MDM4

(49kDa)

PUMA

$\beta$-actin

Ctrl $\mathrm{N}$ Cis Dox Mtx N:C N:D N:M

\section{B}

T778

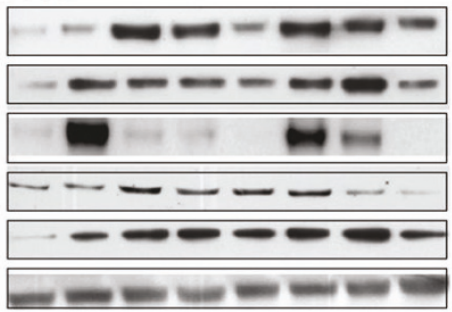

p53

p21

MDM2

MDM4

(49kDa)

PUMA

$\beta$-actin

Ctrl N Cis Dox Mtx N:C N:D N:M

D

\section{RMS13}

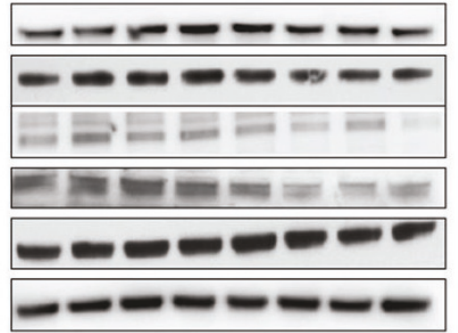

p53

p21

MDM2

MDM4

(49kDa)

PUMA

$\beta$-actin

Ctrl $\mathrm{N}$ Cis Dox Mtx N:C N:D N:M

F
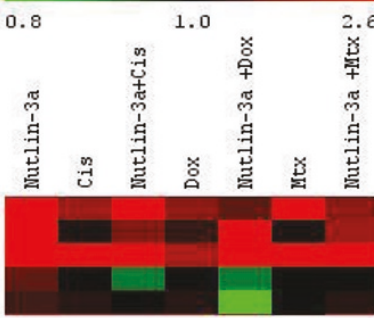

p53

p21

osA

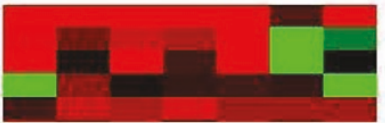

p53

mdm2 T778

$\operatorname{mdm} 4$

puma

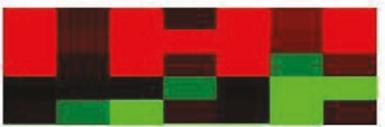

p53

p21 u20s

mdm2

mdm4

puma

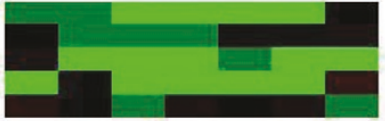

p53

p21

mdm2 RMS13

mom4

p53

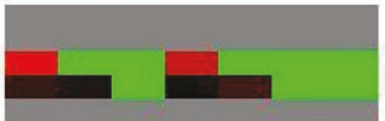

SaOS-2

mdm4

Figure 3 Nutlin-3a modulates MDM2-binding proteins in a p53-dependent manner. Cell lines were exposed to drug-free media (Ctrl), Nutlin-3a (N), Cisplatin (Cis), Doxorubicin (Dox), Methotrexate (Mtx), Nutlin-3a combined with Cisplatin (N:C), Doxorubicin (N:D) or Methotrexate (N:M) in 1:1 ratios. $\mathbf{A}$ and $\mathbf{B}$ illustrate the induction of p53 pathway components by N:C and N:D combinations in the two MDM2 $2^{\mathrm{Ampl}}$ cell lines OSA and T778. C U2OS (MDM2 $\left.{ }^{\text {Wt }}\right)$ displays specific reduction in MDM4 $(62 \mathrm{kDa})$ after Methotrexate exposure alone or in combination with Nutlin3a. $\mathbf{D}$ and $\mathbf{E}$ display the TP53 ${ }^{\text {Mut }}$ cell lines RMS13 and SaOS-2 respectively. The figure shows representative western blots of 3 independent biological experiments, targeting p53, p21, MDM2, MDM4, PUMA, and $\beta$-actin (loading control). Figure $\mathbf{F}$ illustrates quantitative measurements of the same three western blot experiments, values representing mean fold induction ranging from 0.8 (green) to 2.6 fold (red). 
(RMS13), could not be explained by this and clearly needs further investigation.

\section{Conclusions}

Our in vitro data suggest that MDM2 antagonists could offer effective therapy for sarcoma either as single agent or in combination with prevailing chemotherapy, and may significantly reduce the genotoxic burden with the same or better antitumour effect. Further work with animal models of sarcoma and ultimately clinical studies needs to be done to evaluate and optimize combinations of Nutlin and current chemotherapies. Since clear biomarkers are still lacking, clinical trials should be followed up with detailed tumour profiling.

\section{Acknowledgements}

We thank Russell Castro and Heidi M. Namløs for excellent technical assistance and Dr. Anne L. Jacob for useful input during the project planning. The work was supported by the Norwegian Cancer Society and Donations to the Norwegian radium Hospital.

\section{Author details}

'Department of Tumour Biology, The Norwegian Radium Hospital, Oslo University Hospital, P O Box 4953 Nydalen, NO-0424 Oslo, Norway. ${ }^{2}$ Department of Cancer Prevention, The Norwegian Radium Hospital, Oslo University Hospital, P O Box 4953 Nydalen, NO-0424 Oslo, Norway. ${ }^{3}$ Center for Cancer Biomedicine, University of Oslo, 0316 Oslo, Norway. ${ }^{4}$ Roche Research Center, Hoffmann-La Roche Inc., Nutley, NJ 07110, USA. ${ }^{5}$ Institute for Molecular Bioscience, University of Oslo, 0316 Oslo, Norway.

\section{Authors' Contributions}

$\mathrm{HOO}$ carried out the drug sensitivity assays and the combination analysis, performed the statistical analysis and drafted the manuscript. EBP carried out the immunoassays. PN participated in the design of the study and drug sensitivity assays. MB and RAL carried out the sequence analysis. LTV participated in the study design and helped to draft the manuscript. OM conceived the study, participated in its design and coordinated and helped to draft the manuscript. All authors read and approved the final manuscript.

\section{Competing Interests}

Lyubomir Vassilev is employed by Roche, the company which developed Nutlins, and as such could be perceived to have a vested interest in these results. This has however had no consequences for how the studies were performed and the results interpreted.

Received: 19 September 2010 Accepted: 30 May 2011

Published: 30 May 2011

\section{References}

1. Vogelstein B, Lane D, Levine AJ: Surfing the p53 network. Nature 2000, 408:307-310

2. Momand J, Jung D, Wilczynski S, Niland J: The MDM2 gene amplification database. Nucleic Acids Res 1998, 26:3453-3459.

3. Oliner JD, Kinzler KW, Meltzer PS, George DL, Vogelstein B: Amplification of a gene encoding a p53-associated protein in human sarcomas. Nature 1992, 358:80-83.

4. Pilotti S, Della TG, Lavarino C, Di PS, Sozzi G, Minoletti F, et al: Distinct $\mathrm{mdm} 2 / \mathrm{p} 53$ expression patterns in liposarcoma subgroups: implications for different pathogenetic mechanisms. J Pathol 1997, 181:14-24.

5. Florenes VA, Maelandsmo GM, Forus A, Andreassen A, Myklebost O, Fodstad O: MDM2 gene amplification and transcript levels in human sarcomas: relationship to TP53 gene status. J Natl Cancer Inst 1994, 86:1297-1302.

6. Henriksen J, Aagesen TH, Maelandsmo GM, Lothe RA, Myklebost O, Forus A: Amplification and overexpression of COPS3 in osteosarcomas potentially target TP53 for proteasome-mediated degradation. Oncogene 2003, 22:5358-5361.

7. Helman LJ, Meltzer P: Mechanisms of sarcoma development. Nat Rev Cancer 2003, 3:685-694.

8. Brennan MF, Singer S, Maki RG, O'Sullivan B: Soft tissue sarcoma. In Cancer Principles and Practice of Oncology.. 7 edition. Edited by: DeVita Jr VT, Hellman S, Rosenberg SA. Philadelphia: Lippincott Williams and Wilkins; 2005:1581-1637.

9. Malawer MM, Helman LJ, O'Sullivan B: Bone Sarcoma. In Cancer Principles and Practice of Oncology. Volume 2.. 7 edition. Edited by: DeVita Jr VT, Hellman S, Maki RG, O'Sullivan B. Philadelphia: Lippincott Williams and Wilkins; 2005:1638.

10. Vassilev LT, Vu BT, Graves B, Carvajal D, Podlaski F, Filipovic Z, et al: In vivo activation of the p53 pathway by small-molecule antagonists of MDM2. Science 2004, 303:844-848.

11. Tovar C, Rosinski J, Filipovic Z, Higgins B, Kolinsky $\mathrm{K}$, Hilton $\mathrm{H}$, et al: Small-molecule MDM2 antagonists reveal aberrant p53 signaling in cancer: implications for therapy. Proc Natl Acad Sci USA 2006, 103:1888-1893.

12. Coll-Mulet $L$, Iglesias-Serret $D$, Santidrian AF, Cosialls AM, de FM, Castano E, et al: MDM2 antagonists activate p53 and synergize with genotoxic drugs in B-cell chronic lymphocytic leukemia cells. Blood 2006, 107:4109-4114.

13. Secchiero P, Barbarotto E, Tiribelli M, Zerbinati C, di lasio MG, Gonelli A, et al: Functional integrity of the p53-mediated apoptotic pathway induced by the nongenotoxic agent nutlin-3 in B-cell chronic lymphocytic leukemia (B-CLL). Blood 2006, 107:4122-4129.

14. Jiang M, Pabla N, Murphy RF, Yang T, Yin XM, Degenhardt K, et al: Nutlin-3 protects kidney cells during cisplatin therapy by suppressing Bax/Bak activation. J Biol Chem 2007, 282:2636-2645

15. Patton JT, Mayo LD, Singhi AD, Gudkov AV, Stark GR, Jackson MW: Levels of $\mathrm{HdmX}$ expression dictate the sensitivity of normal and transformed cells to Nutlin-3. Cancer Res 2006, 66:3169-3176.

16. Wade M, Wong ET, Tang M, Stommel JM, Wahl GM: Hdmx modulates the outcome of p53 activation in human tumor cells. J Biol Chem 2006, 281:33036-33044.

17. Popowicz GM, Czarna A, Rothweiler U, Sszwagierczak A, Krajewski M, Weber $L$, et al: Molecular Basis for the Inhibition of p53 by Mdmx. Cell Cycle 2007, 6.

18. Freedman DA, Wu L, Levine AJ: Functions of the MDM2 oncoprotein. Cell Mol Life Sci 1999, 55:96-107.

19. Vassilev LT: MDM2 inhibitors for cancer therapy. Trends Mol Med 2007, 13:23-31.

20. Muller CR, Paulsen EB, Noordhuis P, Pedeutour F, Saeter G, Myklebost O: Potential for treatment of liposarcomas with the MDM2 antagonist Nutlin-3A. Int J Cancer 2007, 121:199-205.

21. Cao C, Shinohara ET, Subhawong TK, Geng L, Woon KK, Albert JM, et al: Radiosensitization of lung cancer by nutlin, an inhibitor of murine double minute 2. Molecular Cancer Therapeutics 2006, 5:411-417.

22. Barbieri E, Mehta P, Chen Z, Zhang L, Slack A, Berg S, et al: MDM2 inhibition sensitizes neuroblastoma to chemotherapy-induced apoptotic cell death. Mol Cancer Ther 2006, 5:2358-2365.

23. Stuhmer $T$, Chatterjee M, Hildebrandt M, Herrmann P, Gollasch $H$, Gerecke $C$, et al: Nongenotoxic activation of the p53 pathway as a therapeutic strategy for multiple myeloma. Blood 2005, 106:3609-3617.

24. Drakos E, Thomaides A, Medeiros LJ, Li J, Leventaki V, Konopleva M, et al: Inhibition of p53-murine double minute 2 interaction by nutlin-3A stabilizes p53 and induces cell cycle arrest and apoptosis in Hodgkin lymphoma. Clin Cancer Res 2007, 13:3380-3387.

25. Tabe $Y$, Sebasigari D, Jin L, Rudelius M, vies-Hill T, Miyake $K$, et al: MDM2 antagonist nutlin-3 displays antiproliferative and proapoptotic activity in mantle cell lymphoma. Clin Cancer Res 2009, 15:933-942.

26. Zheng $T$, Wang J, Song $X$, Meng $X$, Pan $S$, Jiang $H$, et al: Nutlin-3 cooperates with doxorubicin to induce apoptosis of human hepatocellular carcinoma cells through p53 or p73 signaling pathways. J Cancer Res Clin Oncol 2010.

27. Carvajal D, Tovar C, Yang H, Vu BT, Heimbrook DC, Vassilev LT: Activation of p53 by MDM2 antagonists can protect proliferating cells from mitotic inhibitors. Cancer Res 2005, 65:1918-1924.

28. Miyachi M, Kakazu N, Yagyu S, Katsumi Y, Tsubai-Shimizu S, Kikuchi K, et al: Restoration of $\mathrm{p} 53$ pathway by nutlin-3 induces cell cycle arrest and 
apoptosis in human rhabdomyosarcoma cells. Clin Cancer Res 2009, 15:4077-4084.

29. Minotti G, Menna P, Salvatorelli E, Cairo G, Gianni L: Anthracyclines: molecular advances and pharmacologic developments in antitumor activity and cardiotoxicity. Pharmacol Rev 2004, 56:185-229.

30. Siddik ZH: Cisplatin: mode of cytotoxic action and molecular basis of resistance. Oncogene 2003, 22:7265-7279.

31. Perego P, Corna E, De CM, Gatti L, Polizzi D, Pratesi G, et al: Role of apoptosis and apoptosis-related genes in cellular response and antitumor efficacy of anthracyclines. Curr Med Chem 2001, 8:31-37.

32. Pedeutour F, Forus A, Coindre JM, Berner JM, Nicolo G, Michiels JF, et al Structure of the supernumerary ring and giant rod chromosomes in adipose tissue tumors. Genes Chromosomes Cancer 1999, 24:30-41.

33. Roberts WM, Douglass EC, Peiper SC, Houghton PJ, Look AT: Amplification of the gli gene in childhood sarcomas. Cancer Res 1989, 49:5407-5413.

34. Berg M, Agesen TH, Thiis-Evensen E, Merok MA, Teixeira MR, Vatn MH, et al: Distinct high resolution genome profiles of early onset and late onset colorectal cancer integrated with gene expression data identify candidate susceptibility loci. Mol Cancer 2010, 9:100.

35. Keepers YP, Pizao PE, Peters GJ, van Ark-Otte J, Winograd B, Pinedo HM: Comparison of the sulforhodamine B protein and tetrazolium (MTT) assays for in vitro chemosensitivity testing. Eur J Cancer 1991, 27:897-900.

36. Chou TC, Talalay P: Quantitative analysis of dose-effect relationships: the combined effects of multiple drugs or enzyme inhibitors. Adv E,nzyme Regul 1984, 22:27-55.

37. Chou TC: Theoretical basis, experimental design, and computerized simulation of synergism and antagonism in drug combination studies. Pharmacol Rev 2006, 58:621-681.

38. Masuda H, Miller C, Koeffler HP, Battifora H, Cline MJ: Rearrangement of the $\mathrm{p} 53$ gene in human osteogenic sarcomas. Proc Natl Acad Sci USA 1987, 84:7716-7719.

39. Felix CA, Kappel CC, Mitsudomi T, Nau MM, Tsokos M, Crouch GD, et al: Frequency and diversity of $\mathrm{p} 53$ mutations in childhood rhabdomyosarcoma. Cancer Res 1992, 52:2243-2247.

40. Joensuu H, Fletcher C, Dimitrijevic S, Silberman S, Roberts P, Demetri G: Management of malignant gastrointestinal stromal tumours. Lancet Oncol 2002, 3:655-664.

41. Andreassen A, Oyjord T, Hovig E, Holm R, Florenes VA, Nesland JM, et al: p53 abnormalities in different subtypes of human sarcomas. Cancer Res 1993, 53:468-471.

42. Korotchkina LG, Demidenko ZN, Gudkov AV, Blagosklonny MV: Cellular quiescence caused by the Mdm2 inhibitor nutlin-3A. Cell Cycle 2009, 8:3777-3781.

43. Toledo F, Wahl GM: MDM2 and MDM4: p53 regulators as targets in anticancer therapy. Int J Biochem Cell Biol 2007, 39:1476-1482.

44. Hu B, Gilkes DM, Farooqi B, Sebti SM, Chen J: MDMX overexpression prevents p53 activation by the MDM2 inhibitor Nutlin. J Biol Chem 2006, 281:33030-33035.

45. Kitagawa M, Aonuma M, Lee SH, Fukutake S, McCormick F: E2F-1 transcriptional activity is a critical determinant of $\mathrm{Mdm} 2$ antagonistinduced apoptosis in human tumor cell lines. Oncogene 2008, 27:5303-5314

46. Lau LM, Nugent JK, Zhao X, Irwin MS: HDM2 antagonist Nutlin-3 disrupts p73-HDM2 binding and enhances p73 function. Oncogene 2008, 27:997-1003.

47. Michaelis M, Rothweiler F, Klassert D, von DA, Weber K, Fehse B, et al: Reversal of P-glycoprotein-mediated multidrug resistance by the murine double minute 2 antagonist nutlin-3. Cancer Res 2009, 69:416-421.

48. Ambrosini G, Sambol EB, Carvajal D, Vassilev LT, Singer S, Schwartz GK: Mouse double minute antagonist Nutlin-3a enhances chemotherapyinduced apoptosis in cancer cells with mutant p53 by activating E2F1. Oncogene 2007, 26:3473-3481.

49. Peirce SK, Findley HW: The MDM2 antagonist nutlin-3 sensitizes p53-nul neuroblastoma cells to doxorubicin via E2F1 and TAp73. Int J Oncol 2009, 34:1395-1402.

50. Banerjee D, Mayer-Kuckuk P, Capiaux G, Budak-Alpdogan T, Gorlick R, Bertino JR: Novel aspects of resistance to drugs targeted to dihydrofolate reductase and thymidylate synthase. Biochim Biophys Acta 2002, 1587:164-173.
51. DeGregori J, Kowalik T, Nevins JR: Cellular targets for activation by the E2F1 transcription factor include DNA synthesis-and G1/S-regulatory genes. Mol Cell Biol 1995, 15:4215-4224.

52. Aslanian A, laquinta PJ, Verona R, Lees JA: Repression of the Arf tumor suppressor by E2F3 is required for normal cell cycle kinetics. Genes Dev 2004, 18:1413-1422.

53. Francoz $S$, Froment $P$, Bogaerts $S$, De CS, Maetens M, Doumont $G$, et al: Mdm4 and Mdm2 cooperate to inhibit p53 activity in proliferating and quiescent cells in vivo. Proc Natl Acad Sci USA 2006, 103:3232-3237.

54. Maguire M, Nield PC, Devling T, Jenkins RE, Park BK, Polanski R, et al: MDM2 regulates dihydrofolate reductase activity through monoubiquitination. Cancer Res 2008, 68:3232-3242.

\section{Pre-publication history}

The pre-publication history for this paper can be accessed here: http://www.biomedcentral.com/1471-2407/11/211/prepub

doi:10.1186/1471-2407-11-211

Cite this article as: Ohnstad et al:: MDM2 antagonist Nutlin-3a potentiates antitumour activity of cytotoxic drugs in sarcoma cell lines. BMC Cancer 2011 11:211.

\section{Submit your next manuscript to BioMed Central and take full advantage of:}

- Convenient online submission

- Thorough peer review

- No space constraints or color figure charges

- Immediate publication on acceptance

- Inclusion in PubMed, CAS, Scopus and Google Scholar

- Research which is freely available for redistribution

Submit your manuscript at www.biomedcentral.com/submit
Biomed Central 